\title{
A Review of the Reliability of Integrated IR Laser Diodes for Silicon Photonics
}

\author{
Matteo Buffolo ${ }^{1, * \mathbb{D}}$, Carlo De Santi ${ }^{1} \mathbb{D}$, Justin Norman ${ }^{2}$, Chen Shang ${ }^{2}$, John Edward Bowers ${ }^{2} \mathbb{D}$, \\ Gaudenzio Meneghesso ${ }^{1}$ D, Enrico Zanoni ${ }^{1}$ and Matteo Meneghini ${ }^{1}$ (D)
}

1 Department of Information Engineering, University of Padova, Via Gradenigo 6/b, 3513 Padova, Italy; carlo.desanti@unipd.it (C.D.S.); gauss@dei.unipd.it (G.M.); zanoni@dei.unipd.it (E.Z.); matteo.meneghini@unipd.it (M.M.)

2 Department of Electrical and Computer Engineering, University of California, Santa Barbara (UCSB), Santa Barbara, CA 93106, USA; jcnorman223@gmail.com (J.N.); shang00@ucsb.edu (C.S.); bowers@ece.ucsb.edu (J.E.B.)

* Correspondence: matteo.buffolo@dei.unipd.it; Tel.: +39-049-827-7625

check for

updates

Citation: Buffolo, M.; De Santi, C.; Norman, J.; Shang, C.; Bowers, J.E.;

Meneghesso, G.; Zanoni, E.;

Meneghini, M. A Review of the Reliability of Integrated IR Laser Diodes for Silicon Photonics. Electronics 2021, 10, 2734. https:// doi.org/10.3390/electronics10222734

Academic Editors: Je-Hyeong Bahk and Yuning $\mathrm{Li}$

Received: 15 September 2021

Accepted: 29 October 2021

Published: 9 November 2021

Publisher's Note: MDPI stays neutral with regard to jurisdictional claims in published maps and institutional affiliations.

Copyright: (c) 2021 by the authors. Licensee MDPI, Basel, Switzerland. This article is an open access article distributed under the terms and conditions of the Creative Commons Attribution (CC BY) license (https:// creativecommons.org/licenses/by/ $4.0 /)$.

\begin{abstract}
With this review paper we provide an overview of the main degradation mechanisms that limit the long-term reliability of IR semiconductor lasers for silicon photonics applications. The discussion is focused on two types of laser diodes: heterogeneous III-V lasers bonded onto silicon-on-insulator wafers, and InAs quantum-dot lasers epitaxially grown on silicon. A comprehensive analysis of the reliability-oriented literature published to date reveals that state-of-the-art heterogeneous laser sources share with conventional laser diodes their major epitaxy-related degradation processes, such as the generation of non-radiative recombination centers or dopant diffusion, while eliminating cleaved facets and exposed mirrors. The lifetime of InAs quantum dot lasers grown on silicon, whose development represents a fundamental step toward a fully epitaxial integration of future photonic integrated circuits, is strongly limited by the density of extended defects, mainly misfit dislocations, protruding into the active layer of the devices. The concentration of such defects, along with inefficient carrier injection and excessive carrier overflow rates, promote recombinationenhanced degradation mechanisms that reduce the long-term reliability of these sources. The impact of these misfits can be largely eliminated with the inclusion of blocking layers.
\end{abstract}

Keywords: silicon photonics; heterogeneous lasers; InAs quantum-dots; degradation; dislocations

\section{Introduction}

Silicon represents the most widely employed semiconductor material for the realizations of electronic devices and integrated circuits. Owing to its indirect bandgap, however, its role in optoelectronics is limited to applications that leverage its waveguiding and modulation properties, rather than its inefficient light emission, or that take advantage of it as a cheap and readily available substrate for the epitaxial growth of other alloys. This means that while high performance detectors and solar cells sensitive in the visible-to near-infrared (NIR) range can be manufactured through well-established Si processes adopted for large-scale microelectronics, the fabrication of light emitters, such as laser diodes and LEDs, has to rely on other semiconductor materials systems, depending on the wavelength of interest. This requirement pushed both the scientific community and companies to develop optimized III-V and II-VI processes and devices that allow the realization of a wide variety of optical sources and sensors. The wavelength of operation of these devices can range from the deep UV, by leveraging the AlGaN/GaN semiconductor material system [1], to the far infrared, for devices based on binary semiconductors such as $\mathrm{GaAs}$, InSb, CdTe, GaP, InAs, and their alloys. While this approach represents the optimal path for the manufacturing of stand-alone components to be included in discrete-based systems, it severely limits the direct inclusion of optical emitters into Si-based electronic 
and photonic circuits [2], which would allow fabricating low-cost fully integrated electrophotonic circuits for a variety of silicon photonics applications. In fact, while efficient and reliable III-N visible LEDs can be grown on silicon substrates despite the presence of lattice mismatch-related extended defects [3-5], continuous-wave (CW) laser operation of IR semiconductor devices requires very-low defect densities to be present within the active region of the devices. Several strategies have been studied and implemented to achieve epitaxial integration of III-V materials on silicon [6-8], but none of these ensured high enough efficiency and scalability to promote their adoption for large-scale integration into Si- and SOI-based photonic and electronic circuits.

Modern PICs for telecom applications circumvent the intrinsic limitation of Si as an optical emitter either by relying on separate laser sources placed in the same package or fiber coupled to the photonic circuit [9], or by selectively bonding III-V laser sources, grown through a dedicated III-V process, onto the SOI substrate by means of low temperature wafer bonding techniques [10-12] (Figure 1). This approach leverages both the high quality of an optical source manufactured through an optimized process and the relatively low manufacturing costs of PICs manufactured through a conventional SOI process $[13,14]$. While this approach is beneficial from a reliability standpoint, since high semiconductor quality implies lower degradation rates and higher robustness against catastrophic optical damages, it is not ideal in terms of industrialization, due to the additional costs and manufacturing complexity associated with the required wafer bonding steps.

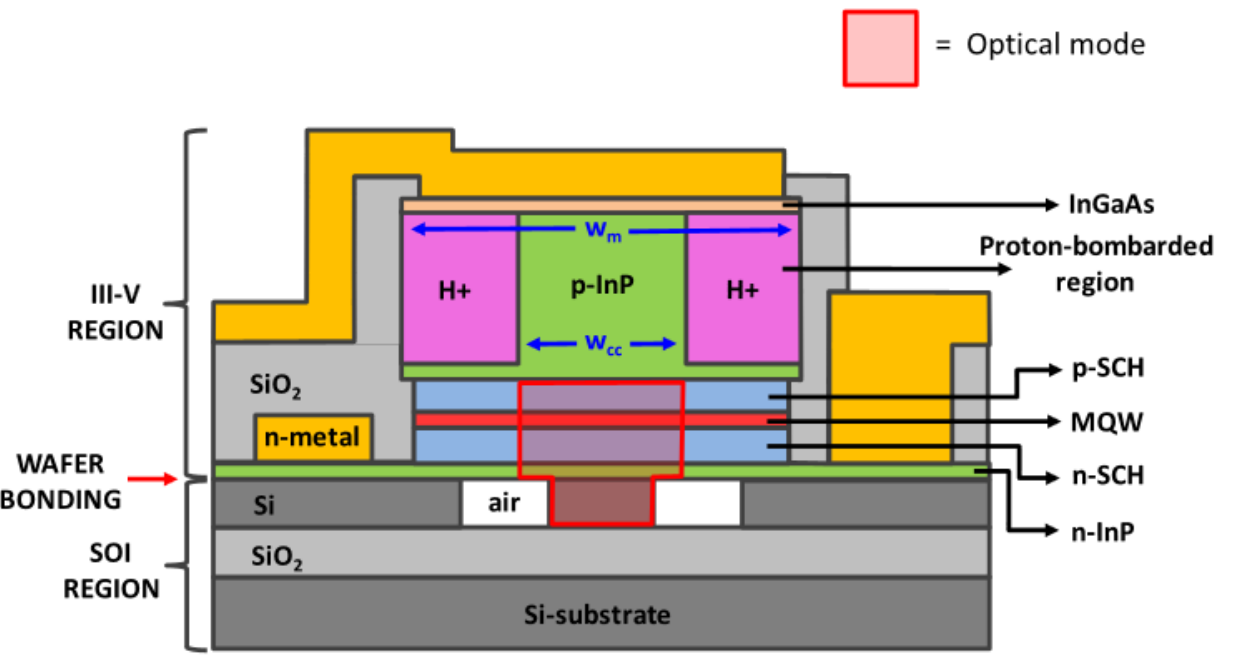

Figure 1. Cross-sectional view of a heterogeneous laser diode (LD): the top III-V part is selectively bonded onto the SOI wafer. The red shaded area highlights the optical mode within the heterogeneous structure. (C) 2017 IEEE. Reprinted, with permission, from M. Buffolo et al., "Degradation Mechanisms of Heterogeneous III-V/Silicon $1.55 \mu \mathrm{m}$ DBR Laser Diodes," in IEEE Journal of Quantum Electronics, Volume 53, No. 4, pp. 1-8, August 2017, Art No. 8400108, doi:10.1109/JQE.2017.2714582 [15].

The most promising alternative to the heterogeneous integration of III-V laser into an SOI process is represented by the adoption of laser diodes based on epitaxial growth of quantum-dots (QDs), rather than on multi-quantum-wells (MQWs) active regions (Figure 2). Owing to their 3D carrier confinement properties [16], the zero-dimensionality QD structures are more tolerant with respect to the presence of extended defects $[17,18]$, and allow the fabrication of reliable and efficient optical sources even in the presence of high densities of dislocations, as in the case of the heteroepitaxial growth of III-V materials onto Si substrates. This fundamental property allowed $1.3 \mu \mathrm{m}$ InAs quantum-dot lasers epitaxially grown on Si with a threading dislocation density (TDD) of $10^{8} \mathrm{~cm}^{-2}$ to reach the lasing threshold and operate continuously, even at high temperature [19]. Once a higher degree of maturity is reached, these devices will allow a fully-epitaxial integration of the optical source into the SOI-based PIC. 


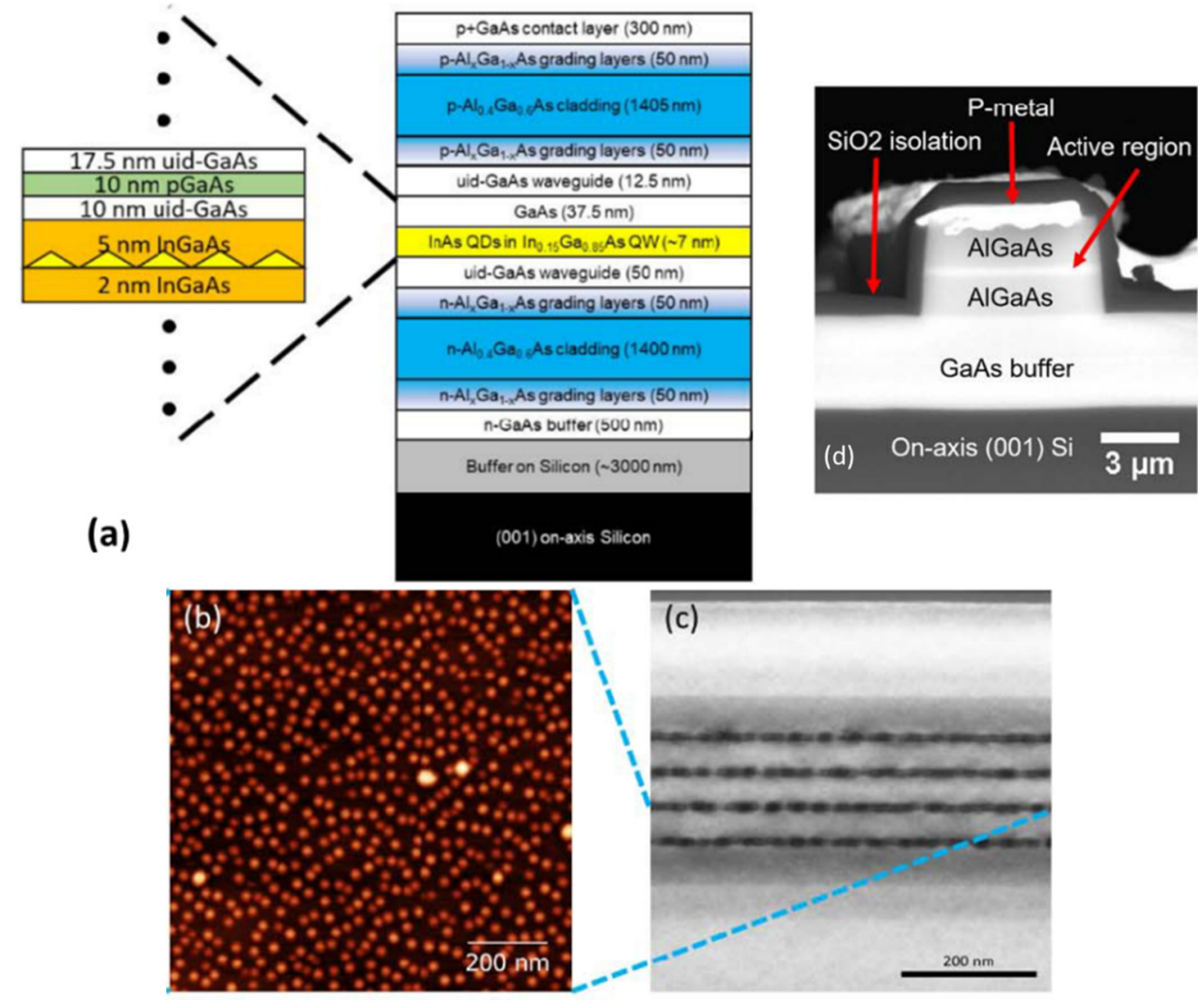

Figure 2. (a) Schematic illustration of the epitaxial structure of a typical InAs quantum-dot laser epitaxially grown on silicon. The figure highlights one period of the active region, which contains a thin p-doped layer within a GaAs barrier, and a single layer of InAs QDs placed within an InGaAs well. (b) Atomic force microscopy (AFM) image of a quantum dot layer: white spots represent InAs quantum-dots. (c) Cross-sectional transmission electron microscope (TEM) image of four layers of quantum dots. (d) Cross-sectional scanning electron microscope (SEM) image of a QD laser facet. (C) 2019 IEEE. Reprinted and adapted, with permission, from J. C. Norman et al., "A Review of High-Performance Quantum Dot Lasers on Silicon," in IEEE Journal of Quantum Electronics, vol. 55, no. 2, pp. 1-11, April 2019, Art no. 2000511, doi:10.1109/JQE.2019.2901508 [16].

Considering that Si-based PICs offer (i) a very high degree of integration, due to the high refractive index contrast between $\mathrm{Si}$ and the surrounding dielectric, (ii) the possibility to integrate all the optical signal conditioning functions required by telecom applications, and (iii) an easy integration with standard CMOS processes and microelectronic circuits, the improvements in the reliability of the integrated optical source represents a fundamental step for the future development of this technology. The aim of this review paper is therefore to describe the device-specific degradation modes and mechanisms determining the long-term reliability of laser diodes for silicon photonics communication applications. In particular, our analysis focuses on heterogeneous III-V MQW lasers on SOI, representing current state-of-the-art integrated lasers, and on quantum-dot laser epitaxially grown on silicon, which may become the preferential light source for future photonic devices and systems operating in the $1.31 \mu \mathrm{m}-1.55 \mu \mathrm{m}$ optical windows.

\section{Reliability of Heterogeneous III-V Lasers on SOI Substrates}

The III-V epitaxies adopted for the fabrication of heterogeneous lasers on SOI substrates do not differ from the ones adopted for conventional laser diodes and therefore, in principle, should exhibit similar degradation modes and mechanisms. During the early stages of development of this novel technology, however, this assumption was not valid, since the additional processing steps required to bond the III-V and the SOI wafers were 
found to cause the formation of thermally-induced defects within the III-V material in correspondence of the bonding interface, as shown in Figure 3 [20].

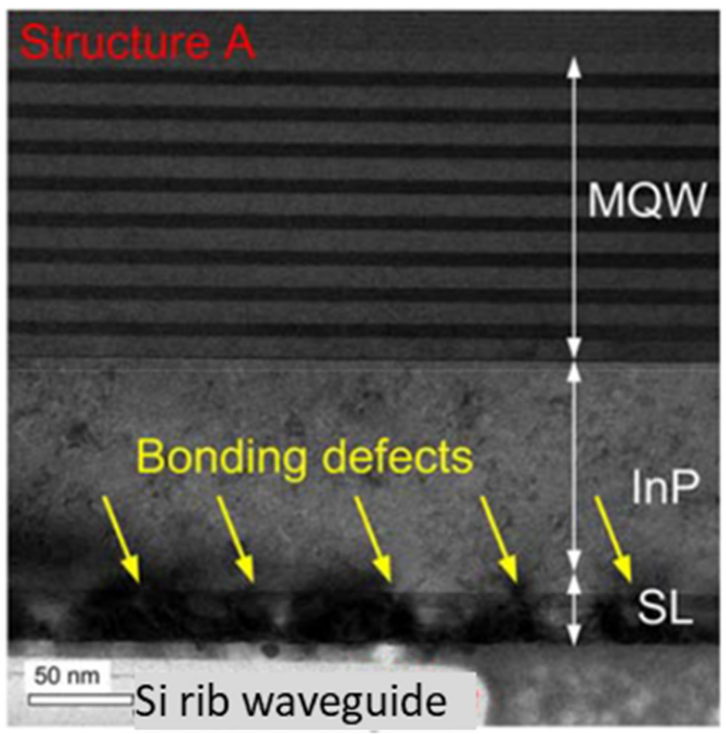

Figure 3. Thermally-induced bonding defects at the interface between the SOI waveguide and the III-V active layer in a heterogeneous laser diode. Image adapted from: D. Liang et al., "Demonstration of Enhanced III-V-On-Silicon Hybrid Integration by Using a Strained Superlattice as a Defect Blocking Layer", ECS Transactions, Volume 33, Number 4, pg. 421-426, 2010, doi:10.1149/1.3483532, (C The Electrochemical Society. Reproduced by permission of IOP Publishing Ltd. All rights reserved [20].

Despite these defects not being associated with the formation of extended defects propagating through the epitaxial layers toward the active region, they were identified to be limiting both device performance and reliability $[20,21]$. To reduce residual lattice strain and avoid the eventual propagation of point defects during device operation, a super-lattice (SL) structure was inserted in the proximity of the bonding interface [22]. The addition of a strained super-lattice effectively increased the performance of the devices measured after the bonding procedure and increased yield from $60 \%$ to $99 \%$ [21]. In addition, the authors found that devices based on epitaxies featuring an SL layer exhibit significantly lower premature failure rates during aging performed at temperature and current density levels up to $85^{\circ} \mathrm{C}$ and $\sim 7 \mathrm{kA} / \mathrm{cm}^{2}$, respectively. Conversely, this optimization did not seem to impact on the degradation rate of non-prematurely failed devices, possibly indicating that their lifetime was mainly determined by other degradation processes. This latter aspect was investigated more in-depth in [15], where the authors evaluated the long-term reliability of $1.55 \mu \mathrm{m}$ distributed Bragg reflector (DBR) heterogeneous laserdiodes by means of a set of constant-current aging experiments carried out at current densities up to $8.6 \mathrm{kA} / \mathrm{cm}^{2}$, at a temperature of $45^{\circ} \mathrm{C}$. In these stress conditions, the heterogeneous laser diodes exhibited a peculiar set of degradation processes, affecting both the optical and electrical characteristics of the devices. In particular, the LDs under investigation showed a gradual and stress-current dependent increase in threshold current that was found to be correlated with the decrease in both turn-on voltage, as in [23], and biasdependent ideality factor, estimated at low forward voltages. These three processes were found to be consistent with the increase in defect concentration within the active region of the device. Conversely, no significant lowering in the slope-efficiency (SE) of the devices was found to affect their optical characteristics. Considering that the slope efficiency of a laser diode can be expressed as [24]:

$$
S E=\eta_{i n j} \frac{\alpha_{m}}{\alpha_{m}+\alpha_{i}}
$$


where $\eta_{\text {inj }}$ is the carrier injection efficiency, and $\alpha_{m}$ and $\alpha_{i}$ are respectively the mirror and the modal losses, this finding indicated that the stress procedure did not impact either the injection efficiency or the mirror losses of the laser. This latter point actually represents a peculiar strength of heterogeneous laser diodes, in terms of reliability. In fact, if a proper optical mode transition between the III-V material and the silicon waveguide is achieved [25-27], the effective facets of the laser can be moved into the optical waveguide and decoupled from the active semiconductor region. This eliminates the problem of facet damage as one of the major sources of device degradation [21], and improves the robustness against catastrophic and gradual mirror degradation [28]. This is important because it enables lower cost, non-hermetic packaging. The central role of the epitaxy, rather than mirrors, in heterogeneous devices degradation was further demonstrated in [29], where the authors showed loop-mirror LDs featuring the same III-V structure of the lasers analyzed in [15] exhibited similar degradation modes.

By analyzing the variation of the capacitance-voltage $(\mathrm{C}-\mathrm{V})$ characteristics during stress, and of the derived apparent charge profile (Figure 4), the authors were able to ascribe the observed gradual increase in threshold current to the relocation of charged defects, or impurities, acting as non-radiative recombination centers (NRRCs). The diffusing species was found to originate from the p-side of the device and to diffuse toward the active region, corresponding in Figure 4 to lower apparent depths $\left(x_{d}\right)$.

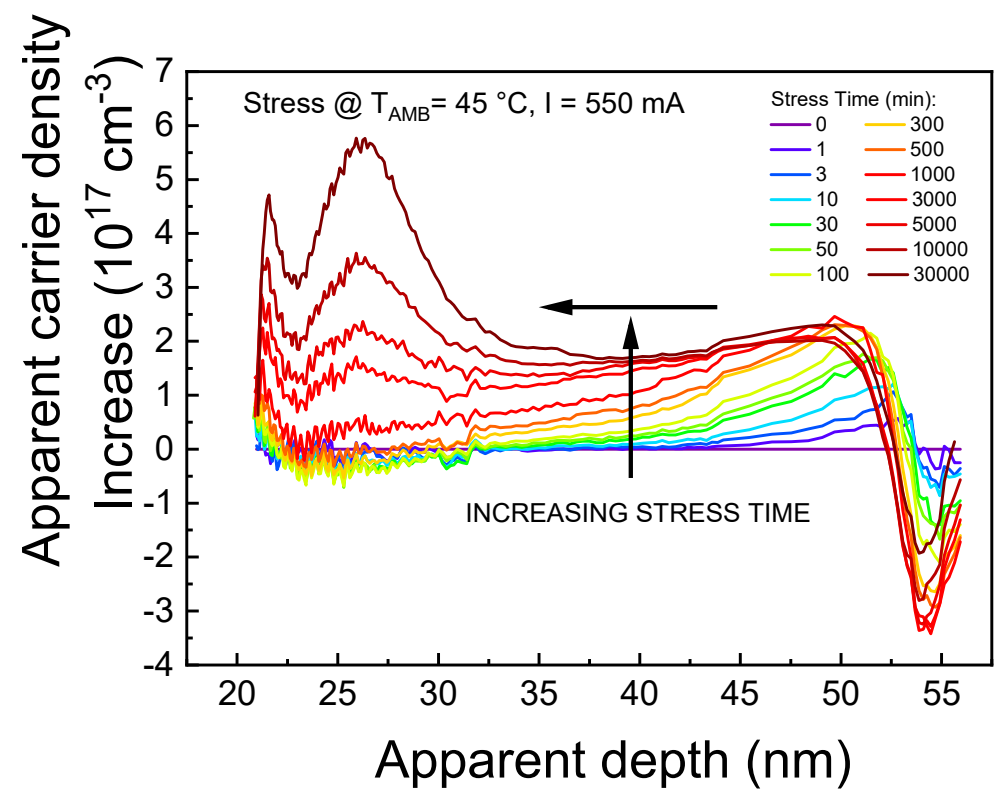

Figure 4. Relative variation of the apparent charge profile, calculated from $\mathrm{C}-\mathrm{V}$ measurements carried out at $1 \mathrm{MHz}$ and $\mathrm{T}=45{ }^{\circ} \mathrm{C}$, of a heterogeneous DBR LD. Variations are referred to the $\mathrm{t}=0$ curve, represented by a flat line. The localized increase in apparent free-charge at specific depths, e.g., at $x_{d}=27 \mathrm{~nm}$, also indicated the presence of physical barriers opposing to the diffusion process. (C) 2017 IEEE. Reprinted, with permission, from M. Buffolo et al., “Degradation Mechanisms

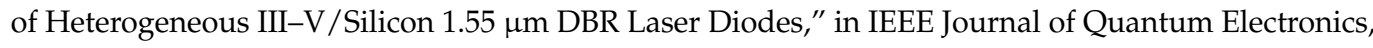
Volume 53, No. 4, pp. 1-8, August 2017, Art No. 8400108, doi:10.1109/JQE.2017.2714582 [15].

In addition to that, by considering that the amount of the observed free charge variation was in the order of $10^{17} \mathrm{~cm}^{-3}$ and that only dopants are intentionally introduced in similar concentrations within the device, the authors postulated that the diffusion of the p-dopant Zn may be driving the observed degradation process. Doping-related diffusion is not something uniquely associated with heterogeneous lasers, since it is inherently related to the III-V epitaxy. This type of degradation can affect both III-N [30] and III-As [31] emitters, and represents the reason why manufacturers of optoelectronic devices tend to transition to less diffusive p-dopants, like carbon, for large scale manufacturing $[32,33]$. Generally speaking, the correlation between optical degradation and 
the generation/propagation of point defects or impurities suggests that even for modern state-of-the-art devices, this kind of degradation process represents a limiting factor for their long-term reliability.

Heterogeneous laser sources have been commercialized for datacenter products with current production rates of several million transceivers/year [34]. The reliability of these devices is superb in spite of the fact that the transceivers are not hermetically packaged. Figure 5 shows $80^{\circ} \mathrm{C}$ aging with negligible aging over 19,000 h [34]. This illustrates the advantage of silicon facets without III-V material at the facet.

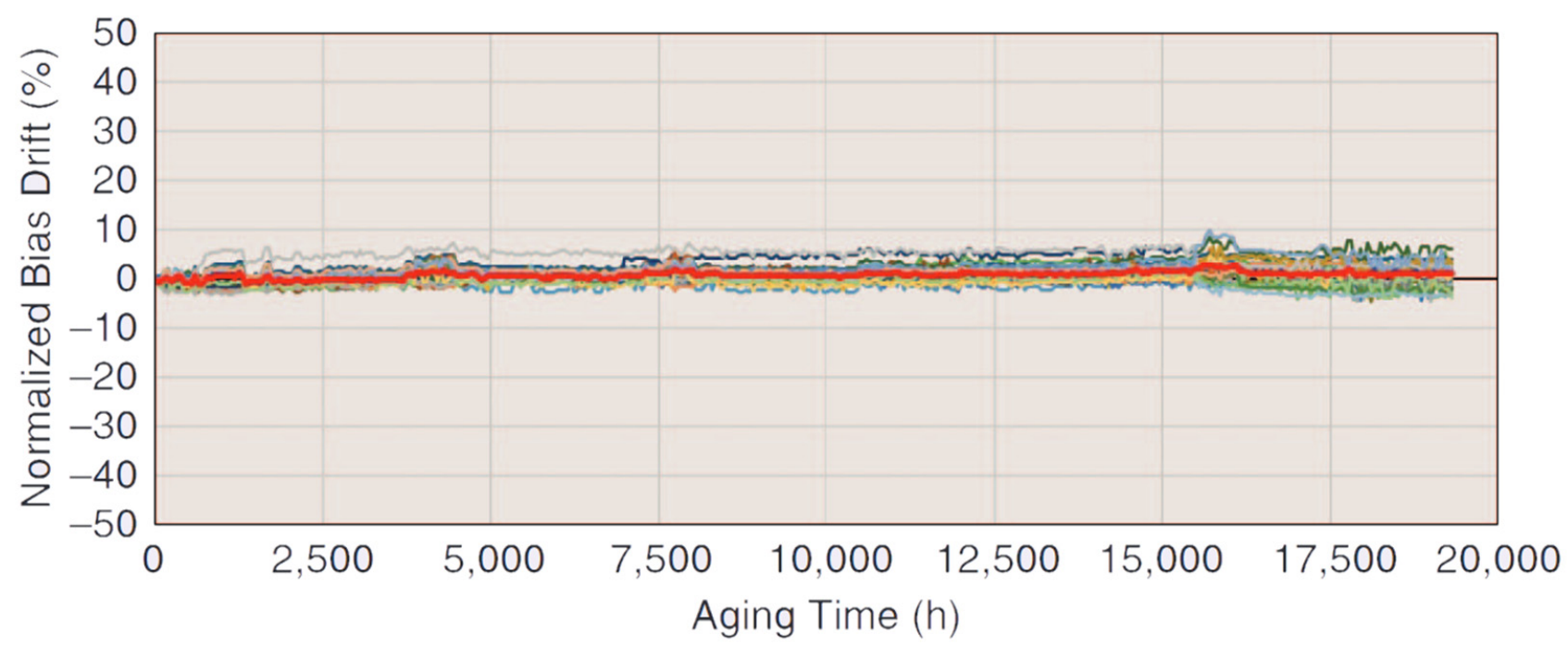

Figure 5. Relative drift of bias current for 10-mW laser output power for $36 \mathrm{III} / \mathrm{V}-\mathrm{Si}$ lasers operating at a stress condition of $80^{\circ} \mathrm{C}$ and I = $150 \mathrm{~mA}$ for over $19 \mathrm{KH}$. (C) 2019 IEEE. Reprinted, with permission, R. Jones et al., "Heterogeneously Integrated InP $\backslash$ /Silicon Photonics: Fabricating Fully Functional Transceivers," in IEEE Nanotechnology Magazine, vol. 13, no. 2, pp. 17-26, April 2019, doi:10.1109/MNANO.2019.2891369 [34].

\section{Reliability of InAs Quantum-Dot Laser Epitaxially Grown on Silicon}

From a reliability standpoint, the advantages offered by a QD-based active layer to heteroepitaxially-grown devices were first demonstrated in [35], where an average lifetime is more than 10 times higher than conventional QW lasers grown on the same GaAs-onsilicon was measured. To understand the physical origin of this dramatic improvement, we must consider that in the presence of a high concentration of extended defects, as in the case of heteroepitaxial III-As layers on $\mathrm{Si}$ [36], non-radiative trap-assisted recombination processes are favored. The energy released by these processes can induce a local increase in lattice temperature, thus favoring the generation of additional defects and promoting other non-radiative recombination events [37]. Therefore, the magnitude of this recombinationenhanced (RE) defects-generation process drastically increases in the proximity of extended defects, like dislocation sites. This mechanism was also confirmed by preliminary materiallevel analyses carried out by means of planar-view scanning TEM (PVSTEM), which revealed that misfit dislocations (MDs) located in the proximity of the active layer acquired a helical component after $600 \mathrm{~h}$ RT aging at $2.5 \mathrm{kA} / \mathrm{cm}^{2}$. Since this is a characteristic behavior of dislocation growth by climb processes [38], this finding indicates that the aging procedure induced the re-growth of pre-existent extended defects. This process was further confirmed by more recent investigations carried out on InAs QD LDs grown on $\mathrm{Si}$ with a lower TDD [39], through which the authors proved that misfit dislocations in device layers submitted to high levels of carrier injection during aging undergo RE-climb/growth: this effect is demonstrated by the evident protrusions affecting the MD in the aged device, compared to the unaged one, as reported in Figure 6. 

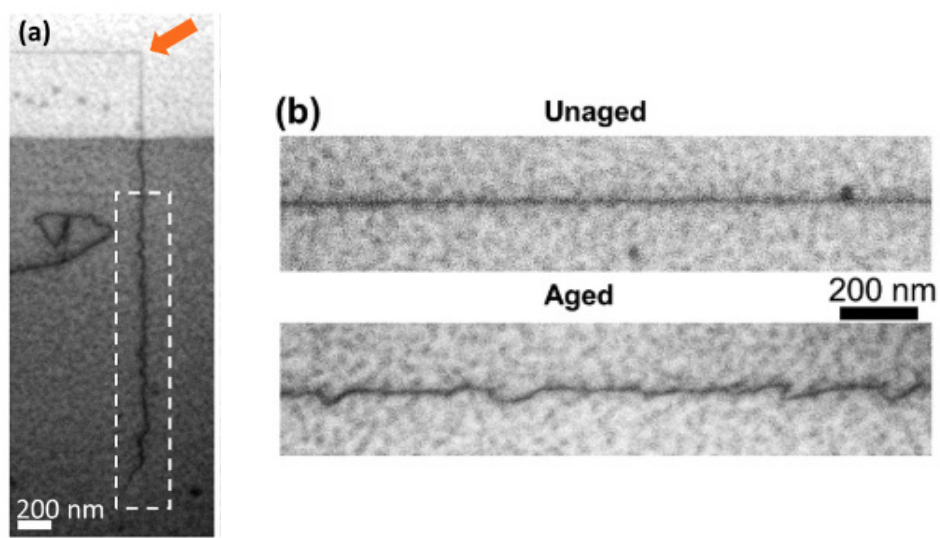

Figure 6. (a) PV-STEM image of a single dislocation exhibiting a misfit segment along 110 direction within an aged QD laser. The lighter (at the top) and darker (at the bottom) regions correspond, respectively, to the device layer submitted to very-low and very-high carrier injection levels. In the un-injected portion of the laser, the misfit dislocation remains straight, whereas dislocation climb occurs in the heavily injected region. (b) PV-STEM of a single misfit dislocation contained within an unaged and an aged QD laser. Evident protrusions are exhibited on one side of the dislocation within the aged device. Reprinted from K. Mukherjee, et al., "Recombination-enhanced dislocation climb in InAs quantum dot lasers on silicon", Journal of Applied Physics 128, 025703 (2020) https: / / doi.org/10.1063/1.5143606, with the permission of AIP Publishing [39].

Since this process is proportional to the number of carriers that are locally captured by the defective sites, the authors explained the huge difference in degradation rate between QD- and QW-based devices by considering that QD structures offer a 3D confinement that spatially localizes injected carrier, thus preventing their capture by nearby extended defective sites (Figure 7). On the other hand, carriers that are not injected into, or that escape from the QDs, are free to laterally diffuse, though with some limitations [40], and promote REDR processes in correspondence of dislocation sites.

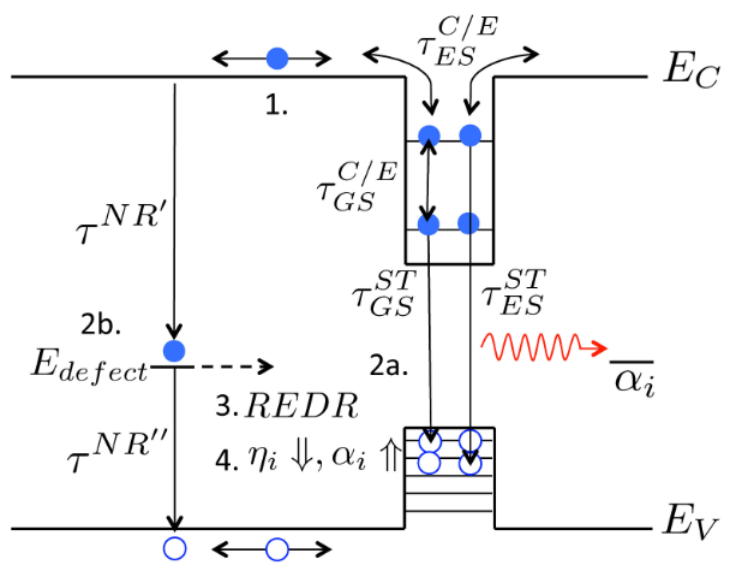

Figure 7. Schematic representation of the RE degradation process occurring in proximity of the active region of InAs QD LDs grown on Si. All the relevant electron-related capture (C), emission (E) and radiative (ST, for stimulated) or non-radiative (NR) recombination processes involved have been highlighted and labeled. Carriers that are injected into the active region (1) can either relax via radiative transitions, if captured by a QD (2a), or via a non-radiative transition, if trapped by a defect (2b). In this second case REDR may lead to growth or formation of new defects (3), inducing the decrease in the internal quantum efficiency and the increase in absorption losses (4). (GS: ground state; ES: excited states). (C) 2015 IEEE. Reprinted, with permission, from A. Y. Liu, et al., "Reliability of InAs/GaAs Quantum Dot Lasers Epitaxially Grown on Silicon," in IEEE Journal of Selected Topics in Quantum Electronics, vol. 21, no. 6, pp. 690-697, Nov.-Dec. 2015, Art no. 1900708, doi:10.1109/JSTQE.2015.2418226 [35]. 
Despite the partial immunity of QD-based LDs to the growth of climb dislocation networks that would usually be seen in comparable QW lasers, it has been demonstrated from a statistical point of view that the density of dislocations still represents a limiting factor for the long-term reliability of InAs QD LDs grown on Si [35]. In particular, a decrease in the TDD of an order of magnitude can increase the device lifetime of a factor approximately equal to 1000, as evidenced by the plots reported in Figure 8.

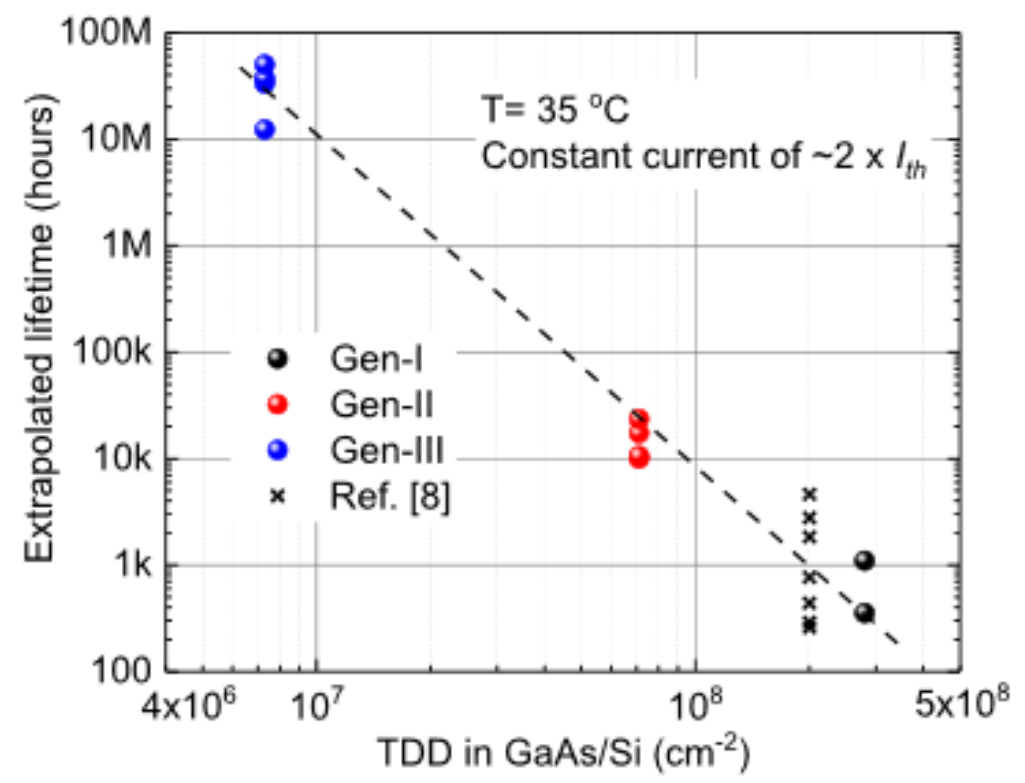

Figure 8. Extrapolated lifetime of quantum-dot lasers grown on silicon with different TDD. Gen I, II, III labels refer to devices grown with a TDD of $2.8 \times 10^{8} \mathrm{~cm}^{-2}, 6 \times 10^{7} \mathrm{~cm}^{-2}$ and $7 \times 10^{6} \mathrm{~cm}^{-2}$, respectively. Reprinted from D. Jung, et al., "Impact of threading dislocation density on the lifetime of InAs quantum dot lasers on Si", Appl. Phys. Lett. 112, 153,507 (2018) https:/ / doi.org/10.1063/1. 5026147, with the permission of AIP Publishing. [41].

The above mentioned findings suggest that lifetime improvements of QD lasers epitaxially grown on silicon should likely proceed through the reduction in the concentration of extended defects, mainly misfit dislocations [39], or through the optimization of the carrier injection dynamics into the InAs QDs [40]. The former goal can either be achieved through the optimization of the growth procedure, of the buffer layer or through a well-engineered positioning of MD-trapping layers that prevent the formation of longitudinally extended defects in the proximity of the active region, which may happen during the heating-up and cooling-down phases of the growth process $[42,43]$. The introduction of misfit trapping layers, which block over $90 \%$ of the misfit dislocations which would otherwise grow along the active region $[43,44]$, allows for a great increase in device reliability, as testified by the higher optical stability exhibited by devices featuring trapping layers within their epitaxy (Figure 9). This misfit growth appears to be the dominant failure mechanism for QD lasers grown on silicon substrates.

On the other hand, a more efficient injection and stimulated recombination of carriers within the QDs can be achieved by adopting a modulation-doping scheme [45], rather than building a conventional p-i-n active structure. This doping approach can favor device performance by easing hole injection, increasing the high-temperature and dynamic performance of QD LDs, and favoring, under appropriate design rules, GS over ES operation [46]. In addition to that, p-modulation doping can improve device reliability, since the faster holes transfer from the modulation doped layers to the GS of the QDs effectively reduces the amount of free carriers available for defect-assisted non-radiative recombination events and degradation processes $[35,41]$. 
Efficient confinement of carriers, and associated device reliability, were also found to exhibit a strong dependence on the operating regime, as evidenced by the experimental results reported in [47].

\begin{tabular}{|c|}
\hline$p$-GaAs contact layer $(300 \mathrm{~nm})$ \\
\hline$p-\mathrm{Al}_{\mathrm{x}} \mathrm{Ga}_{1-\mathrm{x}} \mathrm{As}$ grading layers $(50 \mathrm{~nm})$ \\
\hline$p$ - $\mathrm{Al}_{0.4} \mathrm{Ga}_{0,6} \mathrm{As}$ cladding $(1320 \mathrm{~nm})$ \\
\hline$p$-InGaAs TL $(7 \mathrm{~nm})$ \\
\hline$n-\mathrm{Al}_{04} \mathrm{Ga}_{06} \mathrm{As}$ cladding $(80 \mathrm{~nm})$ \\
\hline$p-\mathrm{Al}_{\mathrm{x}} \mathrm{Ga}_{1-\mathrm{x}} \mathrm{As}$ grading layers $(50 \mathrm{~nm})$ \\
\hline UID-GaAs waveguide $(12.5 \mathrm{~nm})$ \\
\hline p-GaAs $(37.5 \mathrm{~nm})$ \\
\hline InAs QDs in $\ln _{0.15} \mathrm{Ga}_{0.85}$ As QW $~ 7 \mathrm{~nm}$ \\
\hline UID-GaAs waveguide $(50 \mathrm{~nm})$ \\
\hline$n-\mathrm{Al}_{\mathrm{x}} \mathrm{Ga}_{1-\mathrm{x}} \mathrm{As}$ grading layers $(50 \mathrm{~nm})$ \\
\hline$n-\mathrm{Al}_{04} \mathrm{Ga}_{06} \mathrm{As}$ cladding $(80 \mathrm{~nm})$ \\
\hline$n$-InAlAs TL $(7 \mathrm{~nm})$ \\
\hline$n-\mathrm{Al}_{0.4} \mathrm{Ga}_{0,6} \mathrm{As}$ cladding $(1320 \mathrm{~nm})$ \\
\hline$n-\mathrm{Al}_{\mathrm{x}} \mathrm{Ga}_{1-\mathrm{x}} \mathrm{As}$ grading layers $(50 \mathrm{~nm})$ \\
\hline$n$-GaAs grading layers $(50 \mathrm{~nm})$ \\
\hline GaAs/Si template \\
\hline
\end{tabular}

(a)
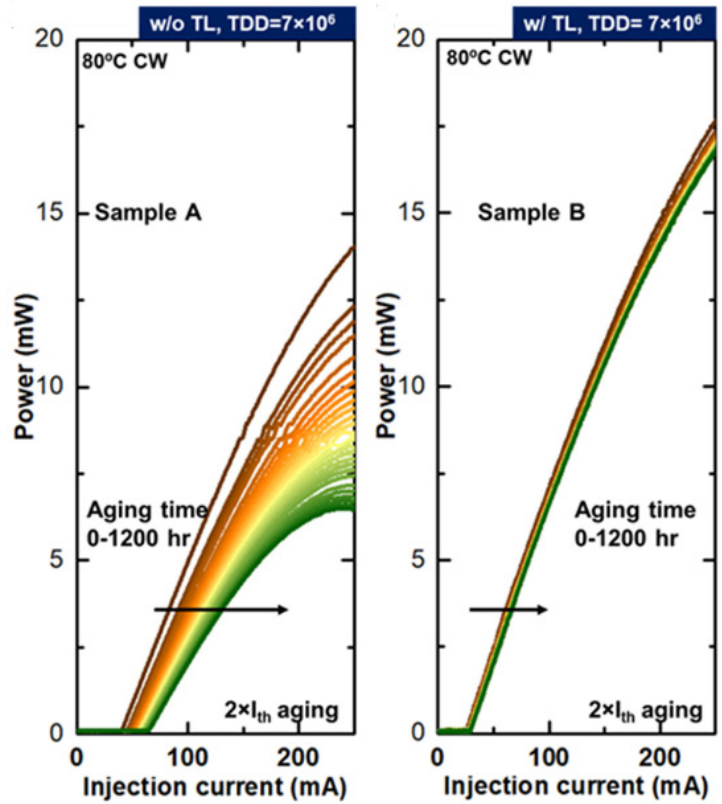

(b)

Figure 9. (a) Epitaxial structure of a QD LDs on Si featuring an MD trapping layer positioned $80 \mathrm{~nm}$ or $180 \mathrm{~nm}$ above and below the active region. (b) Variation of the L-I characteristics during constant-current stress at twice their initial $\mathrm{I}_{\text {th }}$ measured at $80^{\circ} \mathrm{C}$, which also represents the stress temperature. Reprinted with permission from [44] @ The Optical Society.

By means of a current step-stress experiment, carried out at $\mathrm{T}=35^{\circ} \mathrm{C}$ and at current densities up to $10 \mathrm{kA} / \mathrm{cm}^{2}$, the authors were able to ascribe the optical degradation of the QD LDs on Si under test to a defect-related generation process, whose rate was found to increase when the devices are driven at current levels above the onset of ES emission. In this bias regime, corresponding to current levels above $220 \mathrm{~mA}$ (Figure 10), injected carriers tend to occupy QD states at higher energy levels, and therefore see an effective escape barrier which is lower compared to the case of GS-only operation, where most of the carrier occupies the GS of the first ES of the QDs. As a consequence, the rate of escape and of REDR processes described in [35] and by Figure 7 increase, thus increasing the degradation rate. This is also evidenced by the increase in the slope of the $\mathrm{I}_{\mathrm{th}}-1$ /SE curve reported in the right plot of Figure 10 for stress time roughly corresponding to the onset of ES emission. The straight linear correlation between the threshold current increase and the increase in $1 / \mathrm{SE}$ also indicated that the degradation process was lowering the injection efficiency, measured under nominal operating conditions. This kind of variation was found to be compatible with the increase in SRH recombination rate within device layers close to the active region: defect-assisted recombination occurring within these layers prevents carriers from being potentially injected into the GS of the QDs, therefore lowering the apparent injection efficiency of the laser. The effect of non-radiative recombination centers placed within the InGaAs well on the optical performance was also confirmed by numerical simulations, performed on models based on similar device structures [48]. 

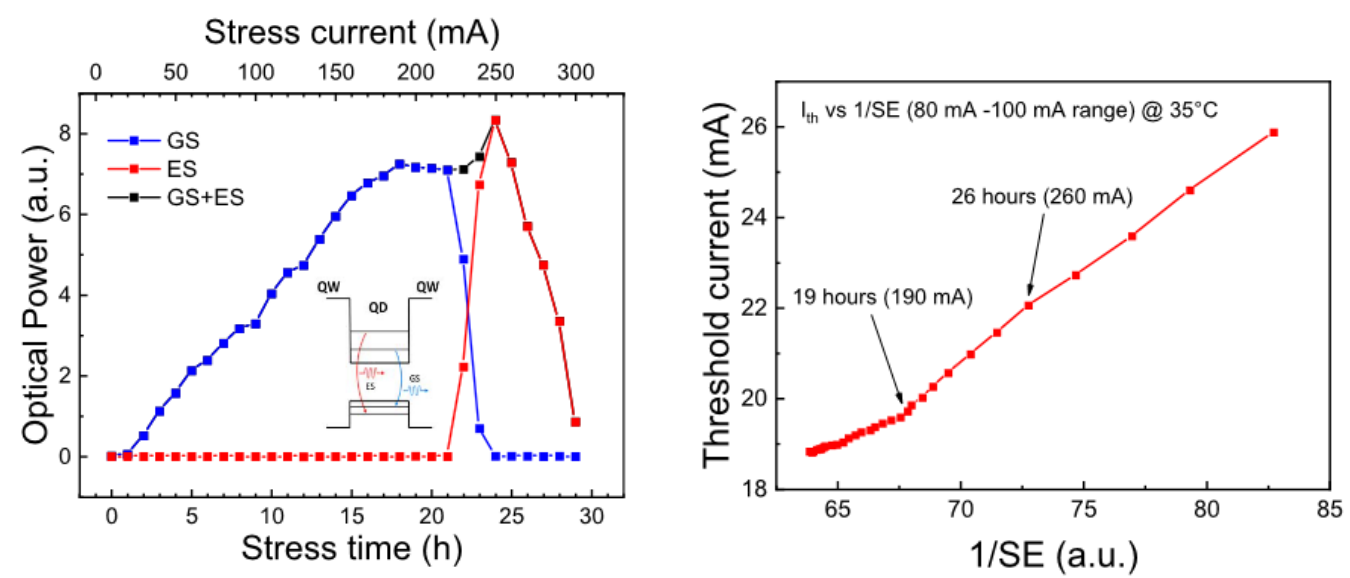

Figure 10. Variation of the optical characteristics of a $3 \times$ QD layer $1.3 \mu \mathrm{m}$ laser grown on $\mathrm{Si}$ with $7 \times 10^{6} \mathrm{~cm}^{-2} \mathrm{TDD}$, submitted to a current step-stress experiment. Left plot: trend of the luminescence from the ground (GS) and excited (ES) states recorded during stress at increasing current. The inset graph shows a sketch of the band-diagram of the DWELL region, showing the transitions related to GS and ES emissions. Right plot: correlation between the variation in threshold current and the change in the reciprocal of slope efficiency. (C) 2019 IEEE. Reprinted, with permission, from M. Buffolo et al., "Physical Origin of the Optical Degradation of InAs Quantum Dot Lasers," in IEEE Journal of Quantum Electronics, Volume 55, No. 3, pp. 1-7, June 2019, Art No. 2000607, doi:10.1109/JQE.2019.2909963 [47].

Further constant-current aging experiments $[49,50]$ confirmed the role of the generation/propagation of non-radiative recombination centers in the gradual optical degradation of QD LDs on Si, evidencing both a strong dependence of this process on stress current density and showing that the observed optical degradation kinetics are compatible with a diffusion process. While this latter finding still requires additional investigations for the determination of the physical origin of the related mechanism, the strong power-law dependence of the degradation rate on stress current density, testified by a mean time-tofailure $(\mathrm{MTTF}) \propto J^{-3.9}$, is compatible with a recombination-driven degradation, since the number of escaping carriers is directly related to the rate of injected carriers during stress.

The improvements in epitaxial quality and the previously described mitigation strategies allowed to attain devices with very-high MTTF. In particular, if we define this latter parameter as the average time required for a sample operating at a constant $10 \mathrm{~mW} \mathrm{OP}$ to increase by two times its initial threshold current, QD LDs with lifetimes in excess of 22 years under high temperature aging $\left(\mathrm{T}=80^{\circ} \mathrm{C}\right)$ have recently been demonstrated [44].

\section{Conclusions}

With this paper, we have reviewed the most relevant scientific results concerning the degradation mechanisms and the reliability of SWIR laser diodes for silicon photonics applications, with a focus on heterogeneous III-V/Si lasers and on InAs quantum-dot lasers epitaxially grown on silicon.

The few available literature reports dealing with the reliability of heterogeneous IR laser diodes indicate that such devices, nowadays, only suffer from minor reliability issues related to the wafer bonding procedure required to couple the III-V IR source to the rest of the photonic circuit contained within the SOI substrate. Additionally, the decoupling of the laser facets from the active semiconductor region increases the robustness against mirror damage and COMD. Conversely, experimental results indicate that these lasers can still be affected by gradual degradation processes involving the generation and/or propagation of impurities and non-radiative recombination centers within the III-V epitaxy. This consideration ultimately shows that point defects may represent a major reliability issue for modern MQW emitters, as far as their long-term reliability is concerned, and suggests that 
a tighter control of defects, as well as the transition to a less diffusive p-type dopant may be necessary steps for the improvement of the lifetime of these novel sources.

On the other hand, statistical analyses, material-oriented investigation and accurate evaluation of the gradual degradation processes related to QD LDs epitaxially grown on $\mathrm{Si}$ evidence that the improvement in the long-term reliability of such devices should proceed mainly through the reduction in dislocation density, mainly misfit-type dislocations, present in correspondence of the active region. The reliability of QD-based lasers is also strongly affected by structural design choices, such as the p-doping approach, that impact on the carrier injection and recombination dynamics within the DWELL region. The optimization of both of these aspects, along with the addition of misfit trapping layers, may allow QD lasers to replace conventional QW-based devices for future silicon photonics applications, and to attain a fully-epitaxial integration of the laser source into the PIC.

Author Contributions: Conceptualization, M.B., C.D.S., J.N., C.S., J.E.B., G.M., E.Z. and M.M.; investigation, M.B., C.D.S., J.N., C.S., J.E.B., G.M., E.Z. and M.M.; resources, M.B., C.D.S., J.N., C.S., J.E.B., G.M., E.Z. and M.M.; data curation, M.B., C.D.S., J.N., C.S., J.E.B., G.M., E.Z. and M.M.; writing-original draft preparation, M.B., C.D.S., J.N., C.S., J.E.B., G.M., E.Z. and M.M.; writingreview and editing, M.B., C.D.S., J.N., C.S., J.E.B., G.M., E.Z. and M.M.; visualization, M.B., C.D.S., J.N., C.S., J.E.B., G.M., E.Z. and M.M.; supervision, M.B., C.D.S., J.N., C.S., J.E.B., G.M., E.Z. and M.M. All authors have read and agreed to the published version of the manuscript.

Funding: This research was supported by INTERNET OF THINGS: SVILUPPI METODOLOGICI, TECNOLOGICI E APPLICATIVI project, co-founded (2018-2022) by the Italian Ministry of Education, Universities and Research (MIUR) under the aegis of the "Fondo per il finanziamento dei dipartimenti universitari di eccellenza" initiative (Law 232/2016).

Conflicts of Interest: The authors declare no conflict of interest. The funders had no role in the design of the study; in the collection, analyses, or interpretation of data; in the writing of the manuscript, or in the decision to publish the results.

\section{References}

1. Amano, H.; Collazo, R.; De Santi, C.; Einfeldt, S.; Funato, M.; Glaab, J.; Hagedorn, S.; Hirano, A.; Hirayama, H.; Ishii, R.; et al. The 2020 UV emitter roadmap. J. Phys. D. Appl. Phys. 2020, 53, 503001. [CrossRef]

2. Paniccia, M. Integrating silicon photonics. Nat. Photonics 2010, 4, 498-499.

3. Lau, K.M. Micro-LED displays: Can the monolithic approach produce full-color? In Proceedings of the Digest of Technical Papers-SID International Symposium, San Jose, CA, USA, May 2019; Blackwell Publishing Ltd.: Hoboken, NJ, USA, 2019; Volume 50, pp. 20-21.

4. Nishikawa, A.; Loesing, A.; Slischka, B. Achieving high uniformity and yield of $200 \mathrm{~mm}$ GaN-on-Si LED epiwafers for micro LED applications with precise strain-engineering. In Proceedings of the Digest of Technical Papers-SID International Symposium, San Jose, CA, USA, May 2019; Blackwell Publishing Ltd.: Hoboken, NJ, USA, 2019; Volume 50, pp. $338-341$.

5. Wang, H.; Lin, Z.; Lin, Y.; Wang, W.; Li, G. High-Performance GaN-Based LEDs on Si Substrates: The Utility of Ex Situ Low-Temperature AlN Template with Optimal Thickness. IEEE Trans. Electron Devices 2017, 64, 4540-4546. [CrossRef]

6. Lee, M.K.; Wuu, D.S.; Tung, H.H. Heteroepitaxial growth of InP directly on Si by low pressure metalorganic chemical vapor deposition. Appl. Phys. Lett. 1987, 50, 1725-1726. [CrossRef]

7. Park, J.S.; Tang, M.; Chen, S.; Liu, H. Heteroepitaxial growth of iii-v semiconductors on silicon. Crystals 2020, 10, 1163. [CrossRef]

8. Sugo, M.; Mori, H.; Tachikawa, M.; Itoh, Y.; Yamamoto, M. Room-temperature operation of an InGaAsP double-heterostructure laser emitting at $1.55 \mu \mathrm{m}$ on a Si substrate. Appl. Phys. Lett. 1990, 57, 593-595. [CrossRef]

9. Buffolo, M.; Meneghini, M.; De Santi, C.; Davenport, M.L.; Bowers, J.E.; Meneghesso, G.; Zanoni, E. Degradation Mechanisms of Heterogeneous III-V/Silicon 1.55- $\mu \mathrm{m}$ DBR Laser Diodes. IEEE J. Quantum Electron. 2017, 53, 1-8. [CrossRef]

10. Margalit, N.; Xiang, C.; Bowers, S.M.; Bjorlin, A.; Blum, R.; Bowers, J.E. Perspective on the future of silicon photonics and electronics. Appl. Phys. Lett. 2021, 118, 220501. [CrossRef]

11. Liang, D.; Roelkens, G.; Baets, R.; Bowers, J.E. Hybrid Integrated Platforms for Silicon Photonics. Materials 2010, 3, 1782-1802. [CrossRef]

12. Liang, D.; Fang, A.W.; Park, H.; Reynolds, T.E.; Warner, K.; Oakley, D.C.; Bowers, J.E. Low-Temperature, $\mathrm{Strong}^{\mathrm{SiO}}{ }_{2}-\mathrm{SiO}_{2}$ Covalent Wafer Bonding for III-V Compound Semiconductors-to-Silicon Photonic Integrated Circuits. J. Electron. Mater. 2008, 37, 1552-1559. [CrossRef]

13. Pasquariello, D.; Hjort, K. Plasma-assisted InP-to-Si low temperature wafer bonding. IEEE J. Sel. Top. Quantum Electron. 2002, 8, 118-131. [CrossRef] 
14. Bowers, J.E.; Liang, D.; Fang, A.W.; Park, H.; Jones, R.; Paniccia, M.J. Hybrid Silicon Lasers: The Final Frontier to Integrated Computing. Opt. Photonics News 2010, 21, 28. [CrossRef]

15. Komljenovic, T.; Davenport, M.; Hulme, J.; Liu, A.Y.; Santis, C.T.; Spott, A.; Srinivasan, S.; Stanton, E.J.; Zhang, C.; Bowers, J.E. Heterogeneous silicon photonic integrated circuits. J. Light. Technol. 2016, 34, 20-35. [CrossRef]

16. Norman, J.C.; Jung, D.; Zhang, Z.; Wan, Y.; Liu, S.; Shang, C.; Herrick, R.W.; Chow, W.W.; Gossard, A.C.; Bowers, J.E. A review of high-performance quantum dot lasers on silicon. IEEE J. Quantum Electron. 2019, 55, 2000511. [CrossRef]

17. Liu, A.Y.; Srinivasan, S.; Norman, J.; Gossard, A.C.; Bowers, J.E. Quantum dot lasers for silicon photonics [Invited]. Photonics Res. 2015, 3, B1. [CrossRef]

18. Selvidge, J.; Norman, J.; Jung, D.; Hughes, E.; Salmon, M.; Bowers, J.; Herrick, R.; Mukherjee, K. Improving Reliability of InAs Quantum Dot Lasers on Silicon Substrates. In Proceedings of the 2019 IEEE Photonics Conference, IPC 2019-Proceedings, San Antonio, TX, USA, 29 September-3 October 2019; Institute of Electrical and Electronics Engineers Inc.: Piscataway, NJ, USA, 2019.

19. Liu, A.Y.; Zhang, C.; Norman, J.; Snyder, A.; Lubyshev, D.; Fastenau, J.M.; Liu, A.W.K.; Gossard, A.C.; Bowers, J.E. High performance continuous wave $1.3 \mu \mathrm{m}$ quantum dot lasers on silicon. Appl. Phys. Lett. 2014, 104, 041104. [CrossRef]

20. Liang, D.; Srinivasan, S.; Peters, J.; Fang, A.; Bowers, J.E. Demonstration of Enhanced III-V-On-Silicon Hybrid Integration by Using a Strained Superlattice as a Defect Blocking Layer. ECS Trans. 2019, 33, 421-426. [CrossRef]

21. Srinivasan, S.; Julian, N.; Peters, J.; Bowers, J.E. Reliability of Hybrid Silicon Distributed Feedback Lasers. IEEE J. Sel. Top. Quantum Electron. 2013, 19, 1501305. [CrossRef]

22. Black, K.A.; Abraham, P.; Karim, A.; Bowers, J.E.; Hu, E.L. Improved luminescence from InGaAsP/InP MQW active regions using a wafer fused superlattice barrier. In Proceedings of the Conference Proceedings. Eleventh International Conference on Indium Phosphide and Related Materials (IPRM'99), Davos, Switzerland, 16-20 May 1999; pp. 357-360. [CrossRef]

23. Fukuda, M.; Iwane, G. Degradation of active region in InGaAsP/InP buried heterostructure lasers. J. Appl. Phys. 1985, 58, 2932-2936. [CrossRef]

24. Coldren, L.A. Diode Lasers and Photonic Integrated Circuits. Opt. Eng. 1997, 36, 616. [CrossRef]

25. Kurczveil, G.; Pintus, P.; Heck, M.J.R.; Peters, J.D.; Bowers, J.E. Characterization of insertion loss and back reflection in passive hybrid silicon tapers. IEEE Photonics J. 2013, 5, 6600410. [CrossRef]

26. Davenport, M.L.; Skendzic, S.; Volet, N.; Hulme, J.C.; Heck, M.J.R.; Bowers, J.E. Heterogeneous silicon/III-V semiconductor optical amplifiers. IEEE J. Sel. Top. Quantum Electron. 2016, 22, 78-88. [CrossRef]

27. Davenport, M.L.; Liu, S.; Bowers, J.E. Integrated heterogeneous silicon/III-V mode-locked lasers. Photonics Res. 2018, 6, 468. [CrossRef]

28. Lambert, R.W.; Ayling, T.; Hendry, A.F.; Carson, J.M.; Barrow, D.A.; McHendry, S.; Scott, C.J.; McKee, A.; Meredith, W. Facetpassivation processes for the improvement of Al-containing semiconductor laser diodes. J. Light. Technol. 2006, 24, 956-961. [CrossRef]

29. Buffolo, M.; Pietrobon, M.; De Santi, C.; Samparisi, F.; Davenport, M.L.; Bowers, J.E.; Zanoni, E.; Meneghini, M. Degradation mechanisms of heterogeneous III-V/Silicon loop-mirror laser diodes for photonic integrated circuits. Microelectron. Reliab. 2018, 88-90, 855-858. [CrossRef]

30. Nam, O.H.; Ha, K.H.; Kwak, J.S.; Lee, S.N.; Choi, K.K.; Chang, T.H.; Chae, S.H.; Lee, W.S.; Sung, Y.J.; Paek, H.S.; et al. Characteristics of GaN-based laser diodes for post-DVD applications. Phys. Status Solidi Appl. Res. 2004, 201, 2717-2720. [CrossRef]

31. Jakubowicz, A.; Oosenbrug, A.; Forster, T. Laser operation-induced migration of beryllium at mirrors of GaAs/AlGaAs laser diodes. Appl. Phys. Lett. 1993, 63, 1185-1187. [CrossRef]

32. Bulaev, P.V.; Marmalyuk, A.A.; Padalitsa, A.A.; Nikitin, D.B.; Zalevsky, I.D.; Konyaev, V.P.; Davydoa, E.I.; Shishkin, V.A.; Sapozhinikov, S.M. Reliability of carbon doped MOCVD grown InGaAs/AlGaAs high power laser diodes. In Proceedings of the CAOL'2003. 1st International Conference on Advanced Optoelectronics and Lasers. Jontly with 1st Workshop on Precision Oscillations in Electronics and Optics, Alushta, Ukraine, 16-20 September 2003; Institute of Electrical and Electronics Engineers (IEEE): Piscataway, NJ, USA, 2004; p. 231.

33. Ayling, S.G.; Bryce, A.C.; Gontijo, I.; Marsh, J.H.; Roberts, J.S. A comparison of carbon and zinc doping in GaAs/AlGaAs lasers bandgap-tuned by impurity-free vacancy disordering. Semicond. Sci. Technol. 1994, 9, 2149-2151. [CrossRef]

34. Jones, R.; Doussiere, P.; Driscoll, J.B.; Lin, W.; Yu, H.; Akulova, Y.; Komljenovic, T.; Bowers, J.E. Heterogeneously Integrated InP/Silicon Photonics: Fabricating fully functional transceivers. IEEE Nanotechnol. Mag. 2019, 13, 17-26. [CrossRef]

35. Liu, A.Y.; Herrick, R.W.; Ueda, O.; Petroff, P.M.; Gossard, A.C.; Bowers, J.E. Reliability of InAs/GaAs quantum dot lasers epitaxially grown on silicon. IEEE J. Sel. Top. Quantum Electron. 2015, 21, 690-697. [CrossRef]

36. Zenari, M.; Buffolo, M.; De Santi, C.; Norman, J.; Meneghesso, G.; Bowers, J.E.; Zanoni, E.; Meneghini, M. Identification of dislocation-related and point-defects in III-As layers for silicon photonics applications. J. Phys. D Appl. Phys. 2021, 54, 8. [CrossRef]

37. Kimerling, L.C. Recombination enhanced defect reactions. Solid State Electron. 1978, 21, 1391-1401. [CrossRef]

38. Petroff, P.; Hartman, R.L. Defect structure introduced during operation of heterojunction GaAs lasers. Appl. Phys. Lett. 1973, 23, 469-471. [CrossRef] 
39. Mukherjee, K.; Selvidge, J.; Jung, D.; Norman, J.; Taylor, A.A.; Salmon, M.; Liu, A.Y.; Bowers, J.E.; Herrick, R.W. Recombinationenhanced dislocation climb in InAs quantum dot lasers on silicon. J. Appl. Phys. 2020, 128, 025703. [CrossRef]

40. Selvidge, J.; Norman, J.; Salmon, M.E.; Hughes, E.T.; Bowers, J.E.; Herrick, R.; Mukherjee, K. Non-radiative recombination at dislocations in InAs quantum dots grown on silicon. Appl. Phys. Lett. 2019, 115, 131102. [CrossRef]

41. Jung, D.; Herrick, R.; Norman, J.; Turnlund, K.; Jan, C.; Feng, K.; Gossard, A.C.; Bowers, J.E. Impact of threading dislocation density on the lifetime of InAs quantum dot lasers on Si. Appl. Phys. Lett. 2018, 112, 153507. [CrossRef]

42. Selvidge, J.; Hughes, E.T.; Norman, J.C.; Shang, C.; Kennedy, M.J.; Dumont, M.; Netherton, A.M.; Zhang, Z.; Herrick, R.W.; Bowers, J.E.; et al. Reduced dislocation growth leads to long lifetime InAs quantum dot lasers on silicon at high temperatures. Appl. Phys. Lett. 2021, 118, 192101. [CrossRef]

43. Selvidge, J.; Norman, J.; Hughes, E.T.; Shang, C.; Jung, D.; Taylor, A.A.; Kennedy, M.J.; Herrick, R.; Bowers, J.E.; Mukherjee, K. Defect filtering for thermal expansion induced dislocations in III-V lasers on silicon. Appl. Phys. Lett. 2020, 117, 122101. [CrossRef]

44. Gossard, A.C.; Shang, C.; Hughes, E.; Selvidge, J.; Bowers, J.E.; Mukherjee, K.; Dumont, M.; Herrick, R.; Koscica, R.; Wan, Y.; et al. High-temperature reliable quantum-dot lasers on Si with misfit and threading dislocation filters. Optica 2021, 8, 749-754. [CrossRef]

45. Norman, J.C.; Zhang, Z.; Jung, D.; Shang, C.; Kennedy, M.J.; Dumont, M.; Herrick, R.W.; Gossard, A.C.; Bowers, J.E. The Importance of p-Doping for Quantum Dot Laser on Silicon Performance. IEEE J. Quantum Electron. 2019, 55, 2001111. [CrossRef]

46. Korenev, V.V.; Savelyev, A.V.; Maximov, M.V.; Zubov, F.I.; Shernyakov, Y.M.; Kulagina, M.M.; Zhukov, A.E. Effect of modulation p -doping level on multi-state lasing in InAs/InGaAs quantum dot lasers having different external loss. Appl. Phys. Lett. 2017, 111, 132103. [CrossRef]

47. Buffolo, M.; Samparisi, F.; De Santi, C.; Jung, D.; Norman, J.; Bowers, J.E.; Herrick, R.W.; Meneghesso, G.; Zanoni, E.; Meneghini, M. Physical Origin of the Optical Degradation of InAs Quantum Dot Lasers. IEEE J. Quantum Electron. 2019, 55, 1-7. [CrossRef]

48. Saldutti, M.; Tibaldi, A.; Cappelluti, F.; Gioannini, M. Impact of carrier transport on the performance of QD lasers on silicon: A drift-diffusion approach. Photonics Res. 2020, 8, 1388. [CrossRef]

49. Buffolo, M.; Samparisi, F.; Rovere, L.; De Santi, C.; Jung, D.; Norman, J.; Bowers, J.E.; Herrick, R.W.; Meneghesso, G.; Zanoni, E.; et al. Investigation of Current-Driven Degradation of $1.3 \mu \mathrm{m}$ Quantum-Dot Lasers Epitaxially Grown on Silicon. IEEE J. Sel. Top. Quantum Electron. 2020, 26, 1-8. [CrossRef]

50. Buffolo, M.; Rovere, L.; De Santi, C.; Jung, D.; Norman, J.; Bowers, J.E.; Herrick, R.W.; Meneghesso, G.; Zanoni, E.; Meneghini, M. Degradation of $1.3 \mu \mathrm{m}$ InAs Quantum-Dot Laser Diodes: Impact of Dislocation Density and Number of Quantum Dot Layers. IEEE J. Quantum Electron. 2020, 57, 1-8. [CrossRef] 